Peter Drgona - Rastislav Stefun - Slavomir Kascak - Jan Morgos

\title{
DEMONSTRATION OF A SYSTEM IDENTIFICATION ON REAL STEP-DOWN POWER CONVERTERS
}

System identification is a scientific field with a wide range of applications, including transport and transportation systems. Automotive industry has a growing trend of power converters implementation. In addition, intelligence of converters is developing. Thus, the power electronics and autotronics are application areas where identification can also be applied. Since buck (step-down), boost (step-up) and buck-boost are the most common topologies of the converters in automobiles, this article aims to demonstrate possibilities of using the identification procedure on the synchronous buck converter. The objective is to obtain a parametric model that could be further useful in analysis and other work with the converter.

Keywords: identification, automotive, buck converter, step-down converter, transfer function

\section{Introduction}

System identification is very interesting nowadays as it can improve and reduce system design time. The aim of identification is to analyze the essential properties and parameters as accurately as possible and to express them in the form of a mathematical model. The best way to analyze a system is to measure it physically, but that is not always possible. During the identification, the parasitic effects and various inaccuracies, neglected in the design, are included, as well, as it is carried out directly on the measured samples from the system. The identification can be used to monitor the condition of the system and its aging, as well as in creation of the adaptive control.

System identification can be implemented also in the power electronics and autotronics, as well, since intelligence in motor vehicles has a growing trend at the present time. Various support systems and electronic assistants are added. They need a stabile voltage to work properly. Therefore, in the current vehicle, one can also find many switched mode power supplies (SMPS), which ensure the supply of electricity to the circuits. These are mainly of the buck, boost, and buck-boost topology. Development of converters is constantly moving forward and intelligent controllers are coming to the forefront. Ability of a converter to recognize its parameters extends the possibilities to improve its dynamic properties and better and more stable control. Ideally, the SMPS regulator should detect changes in the load in real time and adapt its control laws. Such regulators are named as adaptive or self-tuning regulators and should quickly identify their loads, more accurately place poles, better enable transient-suppressing circuits and calculate optimal switching trajectory paths [1].
Methods that aim to determine the transfer function, or the corresponding impulse responses, by direct techniques without selecting a continued set of possible models, are called nonparametric methods. In majority of cases, they are used to obtain the frequency spectrum of the system. Sine-wave testing, or the frequency analysis by the correlation method, take a long time because the methods must be repeated for multiple input frequencies [2]. Identification using the parametric models is motivated by the fact that in many applications and in many situations when one needs to represent a dynamic system in terms of a model, the non-parametric representations, e.g. the Bode plots, Nyquist plots, step responses, etc., are not sufficient. The key step is to select the appropriate model from the model set. A suitable model produces the least possible predictive deviations from the measured data.

In terms of data processing, while minimizing the loss function, one can recognize the one-time identification (offline) methods, where computational operations are performed on a complete data set. The model is created by processing the entire data set. The second group is continuous identification methods (online). They are characterized by a recursive approach to data processing, where the information brought by new - fresh data is only used to correct (update) the original model. The new model is created by repairing the original model [3]. Parameter values estimated using online estimation can vary with time, but parameters estimated using offline estimation do not [4].

In this paper, two experiments on non-isolated digitally controlled DC-DC synchronous step-down converters are presented to demonstrate possibilities of identification. The first converter is C2000 ${ }^{\mathrm{TM}}$ Digital Power BoosterPack from Texas Instruments (TI). Its parameters are: $\mathrm{f}_{\mathrm{SW}}=200 \mathrm{kHz}$,

Peter Drgona, Rastislav Stefun*, Slavomir Kascak, Jan Morgos

Department of Mechatronics and Electronics, Faculty of Electrical Engineering and Information Technology, University of Zilina, Slovakia

*E-mail of corresponding author: rastislav.stefun@feit.uniza.sk 
$\mathrm{L}=4.8 \mu \mathrm{H}, \mathrm{C}_{\mathrm{O}}=396 \mu \mathrm{F}, \mathrm{U}_{\mathrm{IN}}=9 \mathrm{~V}, \mathrm{R}=1.58 \Omega$. The second converter has parameters: $\mathrm{f}_{\mathrm{SW}}=10 \mathrm{kHz}, \mathrm{L}=1 \mathrm{mH}, \mathrm{C}_{\mathrm{O}}=470$ $\mu \mathrm{F}, \mathrm{R}=3.3 \Omega, \mathrm{U}_{\mathrm{IN}}=9 \mathrm{~V}$. Both converters were connected to evaluation kit containing the TMS320F28069F processor for digitally controlling the converter.

\section{Currently used identification methods}

\subsection{Frequency domain data}

Significant work in this area was published by authors of [5-6]. They first started with non-parametric online identification of the step-down converter, then added the forward topology in their work, focused to acquiring characteristics in the frequency domain. They used the cross-correlation method, effectively modified for FPGA circuits. Gradually, it passed from the open loop, where the PRBS signal injected into the fixed duty cycle into the characteristics obtained for the closed-loop control circuit. Very good results in identification field authors obtained also in [6]. Paper deals with parametric online identification implemented on the FPGA circuit. Authors applied the least squares (LS) logarithmic method to the obtained frequency domain data.

\subsection{Recursive least square methods}

Authors used the recursive least square (RLS) method in [1] for online parametric identification of an openloop synchronous step-down converter. Their goal was to monitor the inverter load in real time using a fixed-point processor. Their method can be used for cases where the load stays fixed from the start-up and changes slowly. For example, RLS could identify a battery charger SMPS whose cell count is fixed throughout charging. However, the proposed RLS method, in its most basic form, did not work well for abrupt parameter change, which however, is desired. [1]

In [7], the authors improved the work of authors of [1] in identifying the buck topology converter. The authors designed the fuzzy RLS adaptive method (FRLS). The forgetting factor is continually updated based on the squared prediction error $2(\mathrm{k})$ and the change of squared prediction error $\Delta 2(\mathrm{k})$ using fuzzy logic. Authors simulated the algorithm on both open-loop and closed-loop buck converter. They concluded that the method is relatively simple and is able to estimate accurately parameters during the step load change before the output voltage reaches the maximum allowable value.

In another work [8], the authors again focused on online identification using the RLS using a forgetting factor (referred to as the exponentially weighted least squares method, ERLS). The aim was to create the cheapest and competitive solution for active load identification for the most efficient control of SMPS. The team was the first to apply the dichotomous coordinate descent algorithm
(DCD-RLS) for power electronics in the ERLS method. The DCD is widely used for computationally efficient solution of the system of linear equations, since in the classical RLS approach computationally intensive matrix inversion is required. The authors concluded that the method proposed by them is a suitable alternative to the classical RLS approach in industrial and commercial applications, especially for applications with less powerful processors. The proposed method achieves high accuracy of parameter estimation and low predictive error value. However, compared to conventional RLS techniques, it achieves a slower convergence and is therefore not very suitable for the step load changes.

\subsection{Kalman filter}

Authors proposed in [9] a new parametric system identification method based on a self-tuned Kalman filter (KF). They implemented it into the embedded processor, which controlled the DC-DC synchronous buck converter. In the paper authors concluded that although the RLS methods, especially ERLS, often have satisfactory properties, the KF method is considerably more efficient. It achieves better parameter estimation accuracy and a higher degree of convergence. Moreover, the $\mathrm{KF}$ is less influenced by the measurement noise in the practical implementation compared to the ERLS. The KF method is also stable and does not suffer from a windup effect. The code execution time for the KF was 3 us longer than for the ERLS method. The team also tested the behavior of the algorithm in a step change of load with positive result. The proposed method has potential for practical implementation in real-time applications, especially in adaptive control systems of SMPS [9].

\subsection{Fast affine projection}

In [10], the scientific team applied an algorithm called the fast affine projection (FAP) to a simulated buck topology converter. Affine projection (AP) algorithms are a group of configurable algorithms designed to improve a performance of other adaptive algorithms, especially the least mean square (LMS). The method proposed by the authors achieved very good results compared to the classical RLS or LMS method. The authors achieved the FAP better accuracy, speed of convergence and especially lower computational demands.

\subsection{Steiglitz - McBride algorithm}

Authors led by Peretz researched signal-based identification in the time domain in [11]. Specifically, they applied a step perturbation in the duty cycle command on the buck and the boost converters and sensed output voltage. They used the Steiglitz - McBride algorithm to 


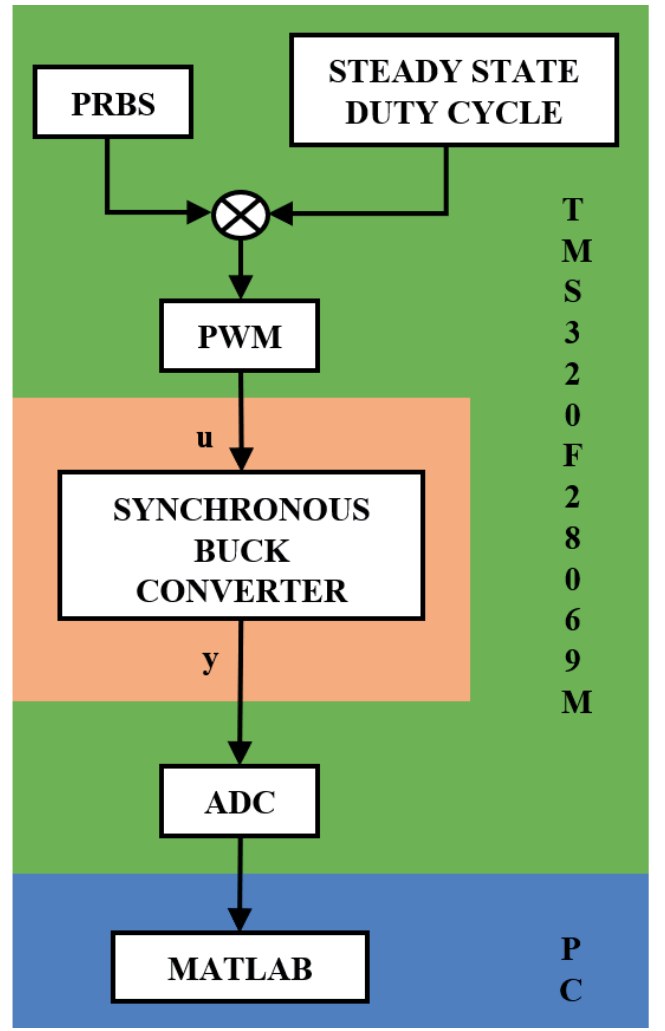

Figure 1 Block schematics of the experiment, where $u$ is input and $y$ is output of the system

determine the system model parameters by iterative the least squares method. The identification procedure was also implemented on digital signal processors (DSP). The method has the advantage that it uses a relatively small number of measured input and output samples sampled at a suitable time. As stated in the paper, the method can be used for direct design of a discrete controller or for determining the output impedance of a converter.

\subsection{Limit cycle oscilations (LCOs)}

Authors that focused on hardware-efficient identification in [12] introduced a method in which the LCOs are used to identify some converter parameters. The method was directly tested on topologies of step-down and step-up converters. The LCOs are an undesirable phenomenon in normal operation but carry useful information about the system. By examining the LCOs amplitude and frequency, it is possible to determine the load R and Q factor. Authors used these parameters for adaptive control and the look up table to speed up the determination of new regulator coefficients. The presented method of identification is also applicable to the low-cost processors, but the method is not very accurate and has many limitations.

\subsection{Component parameters detection}

System identification can also be applied in the field of monitoring the aging of individual components of the converter, or for detecting a fault in the circuit. In a paper [13], authors proposed a method with a non-invasive online way of determining the value of capacity $\mathrm{C}$ and the equivalent series resistance (ESR) of the output capacitor of the buck topology converters. Although the method requires additional circuits, it is very easy to implement. However, it is necessary to know the output inductance of the converter.

\section{Identification verification on real data}

For identification process, it is very important that the input signal meet the excitation stability requirements, because if the system is at a steady state, the information value of the inputs is minimal. Continuous excitation at the system input means that the inputs have such an informative value that the identification algorithm can run smoothly. Otherwise, instability of identification may occur. In practice, this means that the identification algorithm will not be able to capture dynamics of the system and problems of numerical character may occur; exponential convergence of the searched parameters will not be guaranteed. A controlled system becomes unstable, which can lead to serious consequences. Therefore, the pseudo random binary signal (PRBS) was chosen as the excitation signal for the experiment. The PRBS is rectangular pulse sequence modulated in width and appropriately digitally approximates the white noise. It has very similar spectral properties to white noise. It is rich in frequency content. The advantage is also that this signal is periodical and 


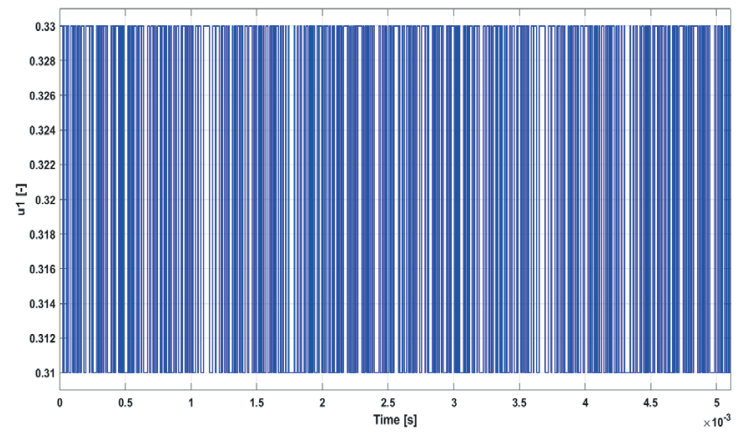

Figure 2 Input signal (u1) of the first converter - duty cycle

deterministic, so that the experiment is repeatable. It is important to choose a sufficiently long period of the PRBS compared to the plant impulse response time [14]. For experiment, 9-bit PRBS with a frequency of $200 \mathrm{kHz}$ or with $10 \mathrm{kHz}$ respectively, were chosen in order not to take up too much memory but at the same time to excite sufficiently the converter. The PRBS frequencies are the same as switching frequencies of the converters. The PRBS had maximum length $\mathrm{L}=511$ and was generated in the $\mathrm{C}$ language by iteratively performing the XOR operation between the 5th and 9th bit. The Block schematics of experiment is shown in Figure 1.

The impulse response of the first converter lasts 0.25 $\mathrm{ms}$ and the actual injections of perturbation lasts for 5.1 $\mathrm{ms}$. The impulse response of the second converter lasts 6 $\mathrm{ms}$ and the actual injections of perturbation lasts for 102.2 ms. Thus, both systems were sufficiently excited. To collect the input and output data a program was written for the processor in CodeComposer.

The input data represents the duty cycle and the output data represents the voltage at the converter output. Two periods of input signal and response to it were sensed. Input and output data were then exported to a PC and analyzed offline in the MATLAB System Identification Toolbox (SIT). Due to the effort to suppress partially the noise, collections of input - output data were repeated 5 times and the data were averaged. The amplitude of the PRBS was set to 0.01 and varied around the steady-state duty cycle value of 0.32 . Thus, the duty cycle values were either 0.31 or 0.33 (Figure 2). This represents a voltage oscillation of $125 \mathrm{~m} \mathrm{~V}$ at the converter output (Figure 3). The steady-state output is 2.04 $\mathrm{V}$, so the perturbation reaches a maximum of $\pm 3 \%$ for both converters. Subsequently, the data were subjected to offline analysis to find a suitable model describing the converter.

In the case of the first converter several models and order variants were tested. The best results were obtained by estimating the state space model of the third order with the noise component $\mathbf{K}$. It represents the noise matrix of the model and it is used for modelling of noise contribution to the model output and for modelling the noise dynamics [4]. Identified discrete-time state-space model is represented by equations and matrices:

$x[n+1]=A x[n]+B u[n]+K e[n]$,

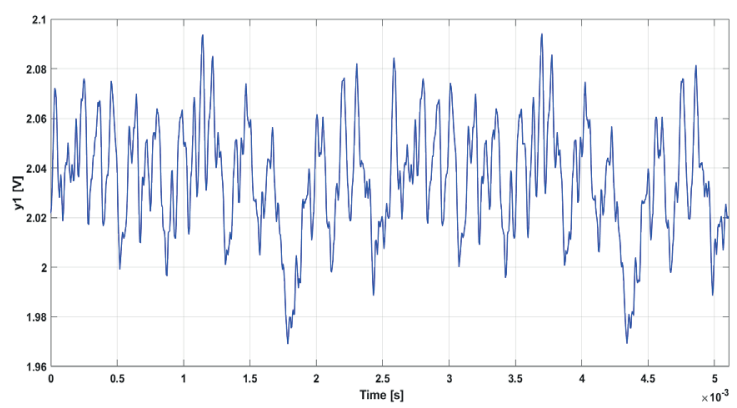

Figure 3 Output signal (u1) of the first converter output voltage

$y[n]=C x[n]+D u[n]+e[n]$,

where the vectors $\mathrm{x}[\mathrm{n}], \mathrm{u}[\mathrm{n}], \mathrm{y}[\mathrm{n}]$ and $\mathrm{e}[\mathrm{n}]$ are the state, input, output and error vectors for the nth sample. State (or system) matrix $\mathbf{A}$, input matrix $\mathbf{B}$, output matrix C, feedthrough (or feedforward) matrix $\mathbf{D}$ and noise component $\mathbf{K}$ are:

$$
\begin{aligned}
& A=\left[\begin{array}{ccc}
0.8772 & 0.4141 & 0.4774 \\
0.00217 & 0.8659 & -0.137 \\
-0.01965 & -0.1107 & 0.6019
\end{array}\right], \\
& B=\left[\begin{array}{c}
0.1686 \\
0.08249 \\
0.6404
\end{array}\right] \text {, } \\
& C=\left[\begin{array}{lll}
1.741 & 0.7932 & -0.3212
\end{array}\right], \\
& D=[0] \text {, } \\
& K=\left[\begin{array}{c}
0.3944 \\
0.1851 \\
-0.03969
\end{array}\right] \text {. }
\end{aligned}
$$

The state space model was consequently converted to control-to-output transfer function using MATLAB command "ss2tf" and it is:

$$
F(z)=\frac{0.1532 z^{-1}+0.3574 z^{-2}-0.4814 z^{-3}}{1-2.3456 z^{-1}+1.802 z^{-2}-0.4524 z^{-3}},
$$

where $z$ is the complex operator of z-transform.

The match of the measured values with the values generated by the model is $90.14 \%$. In comparison, the classical small signal model of the researched converter showed a match of only $61.69 \%$. Identified model was generated by the method of regularized reduction. This method is noniterative. The method works on discrete time-domain data and frequency-domain data. It first estimates a high-order regularized autoregressive model with exogenous inputs (ARX) or finite impulse response (FIR) model, converts it to a state-space model and then performs balanced reduction on it. This method provides improved accuracy on short, noisy data sets [4]. In described experiment, ARX model was used.

It is often necessary to apply a regularization when identifying the higher order models. Regularization is the technique for specifying constraints on a model's flexibility, thereby reducing uncertainty in the estimated parameter values. The kernel is used for regularized estimation of impulse response. The kernel contains information about 


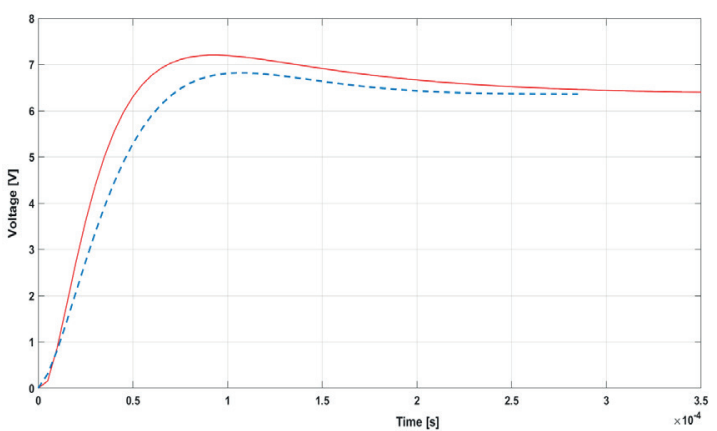

Figure 4 Step response of the first converter - real (blue dashed line) and its identified model (red solid line)

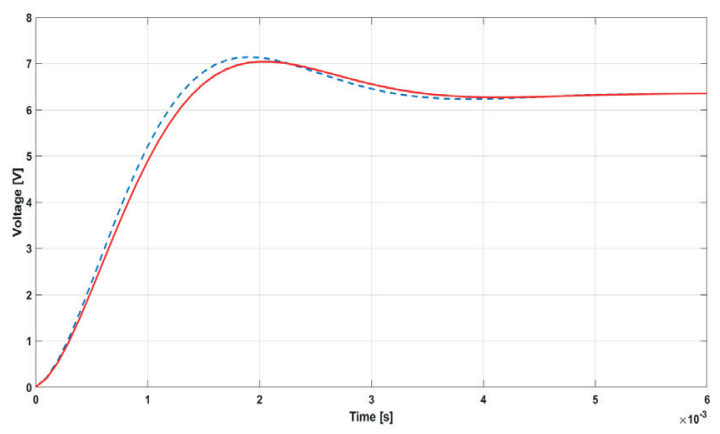

Figure 5 Step response of the second converter - real (blue dashed line) and its identified model (red solid line)

parameterization of the prior covariance of the impulse response coefficients. Regularization reduces variance of estimated model coefficients and produces a smoother response by trading variance for bias. The SIT offers a few options of selection. In the experiment, High frequency (HF) stable spline kernel was chosen as regularization kernel [4].

Identification of the second converter was a simpler task due to fewer passive components present in the converter. Models of the higher orders did not have to be tried. A suitable model was identified directly in the form of the transfer function of the second order:

$T F(z)=\frac{0.00274+0.1893 z^{-1}}{1-1.779 z^{-1}+0.8096 z^{-2}}$.

The match of the measured values with the values generated by the model is $93.55 \%$. The classical small signal model of the researched converter showed a match of $83.18 \%$.

Both identified models are stable - its poles are located inside unit circle. According to [2] it is very important to check not only match of the real data and data produced by the model but the correlations of residuals - differences between the one-step-predicted output from the model and the measured output from the validation data set, as well. Residuals represent the portion of the validation data not explained by the model [4]. Residuals for both converters are inside the confidence interval of the corresponding estimates, indicating that the residuals are uncorrelated.
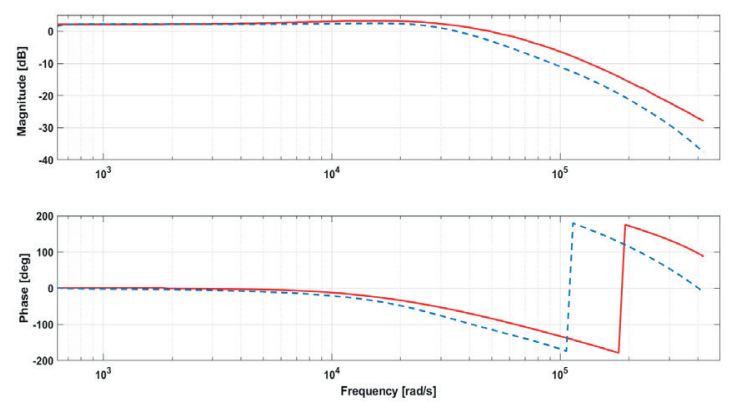

Figure 6 Frequency characteristics of the first converter real (blue dashed line) and its identified model (red solid line)

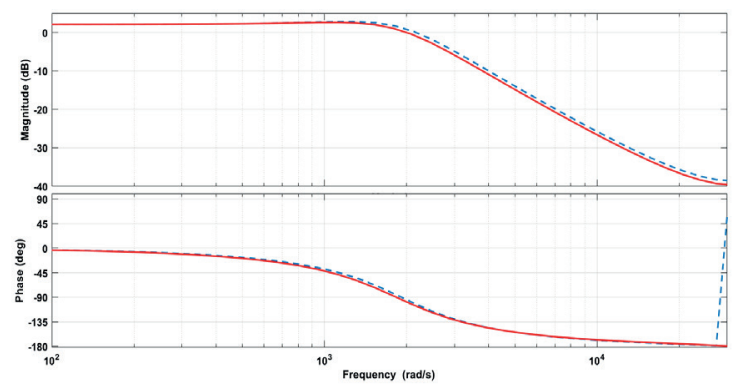

Figure 7 Frequency characteristics of the second converter real (blue dashed line) and its identified model (red solid line)

The identified models were also confronted to the real step-down converters. The step responses of real converters were measured and compared to step responses of identified models. As can be seen in Figures 4 and 5, they are very similar.

The comparison of frequency characteristics is also very important mainly for the control purposes. Software Frequency Response Analyzer (SFRA) library developed by the TI for obtaining frequency characteristics of converters was used to collect frequency response data of real buck converters. It is based on injecting a sinusoidal signal into the duty cycle and measuring the response of the system [15]. Characteristics of the researched converters were compared to the frequency characteristics of the models (Figure 6, Figure 7). They have a high match.

\section{Conclusion}

The article deals with demonstration of identification possibilities applied in the field of power electronics and autotronics. In addition to mapping the currently used methods, the article deals with the offline identification of the real step-down converters. Identification was performed offline on real measured input and output data, which were subsequently processed in MATLAB. Suitable models, describing the converters, were found. In the case of the first converter, model was found in the state-space form of the third order, which was subsequently transformed into a transfer function. The identified model shows a higher 
match with the measured data than the standard small signal model. In the case of the second converter, it was possible to find the simpler model of the second order. Both models have very similar properties for the real step-down converters and can be used as mathematical models of the real converters. Proposed identification procedure could be applied in automotive industry.

\section{Acknowledgment}

Authors would like to thank to APVV-15-0571- Research of the optimum energy flow control in the electric vehicle system.

\section{References}

[1] PITEL, G. E., KREIN, P. T. Real-time system identification for load monitoring and transient handling of DC-DC supplies. In: 39th IEEE Annual Power Electronics Specialists Conference PESC '08:proceedings [online]. 2008. ISSN 0275-9306, p. 3807-3813. Available from: https://doi.org/10.1109/PESC.2008.4592548

[2] LJUNG, L. System identification. 2. ed. USA: PTR Prentice Hall,1999. ISBN 0-13-656695-2.

[3] TAKACS, G., VACHALEK, J., ROHAL-ILKIV, B. System identification / Identifikacia sustav (in Slovak). Bratislava: STU, 2014. ISBN 978-80-227-4288-7.

[4] LJUNG, L. System identification toolbox user's guide. 2014.

[5] MIAO, B., ZANE, R., MAKSIMOVIC, D. (N. D.). Practical on-line identification of power converter dynamic responses. In: 20th Annual IEEE Applied Power Electronics Conference and Exposition APEC 2005: proceedings. 2005.

[6] MIAO, B., ZANE, R., MAKSIMOVIC, D. (N. D.). Automated digital controller design for switching converters. In: IEEE 36th Conference on Power Electronics Specialists: proceedings [online]. IEEE, 2005. ISBN 0-7803-9033-4. Available from: https://doi.org/10.1109/PESC.2005.1582019

[7] AL-GREER, M., ARMSTRONG, M., GIAOURIS, D. System identification of PWM DC-DC converters during abrupt load changes. In: 35th Annual Conference of IEEE Industrial Electronics: proceedings [online]. IEEE, 2009. ISSN 1553-572X, ISBN 9781424446483, p. 1675-1680. Available from: https://doi.org/10.1109/IECON.2009.5414842

[8] AL-GREER, M., ARMSTRONG, M., GIAOURIS, D. active online system identification of switch mode DC-DC power converter based on efficient recursive DCD-IIR adaptive filter. IEEE Transactions on Power Electronics [online]. 2012, 27(11), p. 4425-4435. ISSN 0885-8993, eISSN 1941-0107. Available from: https://doi.org/10.1109/TPEL.2012.2190754

[9] AHMEID, M., ARMSTRONG, M., GADOUE, S., ALGREER, M., MISSAILIDIS, P. Real-time parameter estimation of DC-DC converters using a self-tuned Kalman filter. IEEE Transactions on Power Electronics [online]. 2017, 32(7), p. 5666-5674. ISSN 0885-8993, eISSN 1941-0107. Available from: https://doi.org/10.1109/TPEL.2016.2606417

[10] WANG, CH., MISSAILIDIS, P., GADOUE, S., ARMSTRONG, M. System identification of a DC-DC converter system using a fast affine projection algorithm. In: 7th IET International Conference on Power Electronics, Machines and Drives PEMD 2014: proceedings [online]. IET, 2004. ISBN 978-1-84919-815-8. Available from: https://doi.org/10.1049/ cp.2014.0347

[11] PERETZ, M. M., BEN-YAAKOV, S. Time-domain identification of pulse-width modulated converters. IET Power Electronics [online]. 2012, 5(2), p. 166-172. ISSN 1755-4535. Available from: https://doi.org/10.1049/iet-pel.2010.0377

[12] ZHAO, Z., PRODIC, A. Limit-cycle oscillations based auto-tuning system for digitally controlled DC-DC power supplies. IEEE Transactions on Power Electronics [online]. 2007, 22(6), p. 2211-2222. ISSN 0885-8993, eISSN 1941-0107. Available from: https://doi.org/10.1109/TPEL.2007.909307

[13] YAO, K., TANG, W., HU, W., LYU, J. A current-sensor-less online ESR and C identification method for output capacitor of buck converter. IEEE Transactions on Power Electronics [online]. 2015, 30(12), p. 6993-7005. ISSN 0885-8993, eISSN 1941-0107. Available from: https://doi.org/10.1109/TPEL.2014.2383436

[14] ZAKHAROV, Y. V. Low-complexity implementation of the Affine projection algorithm. IEEE Signal Processing Letters [online]. 2008, 15, p. 557-560. ISSN 1070-9908. Available from: https://doi.org/10.1109/LSP.2008.2001111

[15] BHARDWAJ, M. C2000 software frequency response analyzer (SFRA) library \& compensation designer module user's guide. Dallas, Texas: Texas Instrument, 2015. 